\title{
Does the nuclear spin relaxation rate in superconductors depend on disorder?
}

\author{
K. V. Samokhin ${ }^{1,2}$ and B. Mitrović ${ }^{1}$ \\ 1 Department of Physics, Brock University, St.Catharines, Ontario L2S 3A1, Canada \\ ${ }^{2}$ Commissariat à l'Energie Atomique, DSM/DRFMC/SPSMS, 38054 Grenoble, France
}

\begin{abstract}
We calculate the relaxation rate of a nuclear spin in $s$-wave superconductor with nonmagnetic impurities, including the strong-coupling effects. We show that in a weakly disordered threedimensional system the corrections due to disorder are negligibly small.

PACS numbers: $74.25 . \mathrm{Nf}, 74.20 . \mathrm{-z}$
\end{abstract}

\section{INTRODUCTION}

In this article we revisit a long-standing problem about the effect of nonmagnetic impurities on the nuclear spin relaxation rate $\underline{\underline{1}} R_{s}(T)$ in isotropic superconductors. The first calculation of $R_{s}$ in a clean superconductor using the Bardeen-Cooper-Schrieffer (BCS) model was done by Hebel and Slichter ${ }^{2}$. They noticed that the expression for the relaxation rate is logarithmically divergent at all temperatures below $T_{c}$, due to the singularity of the BCS quasiparticle density of states (DoS) at the gap edge. This singularity survives even in the presence of scalar disorder (since, according to Anderson's theorem, the superconducting gap is not affected by nonmagnetic impurities) and was removed in Ref. $\frac{2}{2}$ by phenomenologically introducing some energy level broadening. A few years later, Maki and Fulde $\mathrm{e}^{\underline{3}}$ calculated the relaxation rate in a superconductor with magnetic impurities, in which case the DoS singularity is smeared and the expression for $R_{s}$ is finite at all $T$. It was shown in Ref. $\frac{3}{\cdot}$ that the impurity vertex corrections to the relaxation rate are negligible. In a parallel development, the Hebel-Slichter arguments about the importance of the level broadening were put on a quantitative footing by Fibich $\stackrel{4}{ }$, see also Ref.$^{\underline{5}}$, who calculated the relaxation rate in the clean case using the Eliashberg theory of electron-phonon superconductivity $\underline{\underline{6}}$ (the so-called strong coupling model) and found that inelastic scattering indeed removes the gap-edge singularity and produces a finite relaxation rate.

The effect of scalar impurities in the strong-coupling regime remained an open problem for another three decades until Choi and Mele ${ }^{7}$ presented a calculation of the relaxation rate, in which they questioned the accuracy of the MakiFulde result and claimed that the impurity vertex corrections are important. As a result, $R_{s}$ appreciably increases with nonmagnetic disorder, especially in the dirty case $\ell \lesssim \xi_{0}$, where $\ell$ is the elastic mean free path and $\xi_{0}$ is the superconducting coherence length. In the present article we try to resolve this controversy. The article is organized as follows: In section II, we calculate the relaxation rate in the weak-coupling BCS limit, using the exact eigenstates method to perform the disorder averaging. In section [III the exact eigenstates method is generalized to the strongcoupling case. In section IV] we calculate the relaxation rate in the strong-coupling case by another method, using a standard disorder averaging technique involving the summation of the ladder impurity diagrams.

\section{EXACT EIGENSTATES METHOD: WEAK COUPLING}

Without loss of generality we consider the case of a nuclear spin $I=1 / 2$ located at the origin of the crystal lattice. Higher values of $I$ change only the overall prefactor in the expression for the relaxation rate $\frac{1}{}$, which drops out of the ratio of the relaxation rates in the superconducting and the normal states. We assume, following Hebel and Slichter ${ }^{2}$ that, while the resonance is observed in a strong field in the normal state, the relaxation takes place in a uniform superconducting state after switching off the field. The spin-lattice relaxation rate due to the hyperfine contact interaction of the nucleus with the band electrons is given by

$$
R \equiv \frac{1}{T_{1} T}=-J^{2} \lim _{\omega_{0} \rightarrow 0} \frac{\operatorname{Im} K^{R}\left(\omega_{0}\right)}{\omega_{0}},
$$

where $J$ is the hyperfine coupling constant, $\omega_{0}$ is the NMR frequency, and $K^{R}$ is the retarded correlator of the transverse components of the electron spin density at the nuclear site ${ }^{1.8}$, which is calculated as follows. We introduce $K\left(\nu_{m}\right)$, with $\nu_{m}=2 \pi m T$, as the Fourier transform of

$$
K(\tau)=-\left\langle\left\langle T_{\tau} S_{+}(\mathbf{0}, \tau) S_{-}(\mathbf{0}, 0)\right\rangle\right\rangle_{i m p} .
$$

Here $\langle\cdots\rangle$ denotes the quantum statistical average and $\langle\cdots\rangle_{i m p}$ denotes the averaging over impurity configurations $s^{9,10}$. The spin density operators in the Matsubara representation are given by $S_{ \pm}(\boldsymbol{r}, \tau)=e^{H \tau} S_{ \pm}(\boldsymbol{r}) e^{-H \tau}$, where $H$ is the 
electron Hamiltonian, to be defined below, and

$$
S_{+}(\boldsymbol{r})=\psi_{\uparrow}^{\dagger}(\boldsymbol{r}) \psi_{\downarrow}(\boldsymbol{r}), \quad S_{-}(\boldsymbol{r})=\psi_{\downarrow}^{\dagger}(\boldsymbol{r}) \psi_{\uparrow}(\boldsymbol{r}) .
$$

We use the units in which $\hbar=k_{B}=1$, and the spin quantization axis is chosen along the external magnetic field $\boldsymbol{H}$. The retarded correlator in Eq. (11) is obtained by the analytic continuation: $K^{R}\left(\omega_{0}\right)=\left.K\left(\nu_{m}\right)\right|_{i \nu_{m} \rightarrow \omega_{0}+i 0}$.

The properties of our system in the superconducting state can be described using a standard field-theoretical formalism in terms of the normal and anomalous Gor'kov functions ${ }^{9}$, which can be combined into a $2 \times 2$ matrix Green's function

$$
\hat{G}\left(\boldsymbol{r}, \tau ; \boldsymbol{r}^{\prime}, \tau^{\prime}\right)=\left(\begin{array}{cc}
G\left(\boldsymbol{r}, \tau ; \boldsymbol{r}^{\prime}, \tau^{\prime}\right) & -F\left(\boldsymbol{r}, \tau ; \boldsymbol{r}^{\prime}, \tau^{\prime}\right) \\
-F^{\dagger}\left(\boldsymbol{r}, \tau ; \boldsymbol{r}^{\prime}, \tau^{\prime}\right) & -G\left(\boldsymbol{r}^{\prime}, \tau^{\prime} ; \boldsymbol{r}, \tau\right)
\end{array}\right)
$$

Introducing the two-component Nambu operators ${ }^{11}$

$$
\Psi(\boldsymbol{r}, \tau)=\left(\begin{array}{c}
\psi_{\uparrow}(\boldsymbol{r}, \tau) \\
\psi_{\downarrow}^{\dagger}(\boldsymbol{r}, \tau)
\end{array}\right) \quad, \quad \bar{\Psi}(\boldsymbol{r}, \tau)=\left(\psi_{\uparrow}^{\dagger}(\boldsymbol{r}, \tau) \quad \psi_{\downarrow}(\boldsymbol{r}, \tau)\right),
$$

the matrix Green's function can be written in a compact form:

$$
\hat{G}\left(\boldsymbol{r}, \tau ; \boldsymbol{r}^{\prime}, \tau^{\prime}\right)=-\left\langle T_{\tau} \Psi(\boldsymbol{r}, \tau) \bar{\Psi}\left(\boldsymbol{r}^{\prime}, \tau^{\prime}\right)\right\rangle
$$

The Matsubara spin correlator (2) can be expressed as the impurity average of the product of two matrix Green's functions:

$$
K\left(\nu_{m}\right)=\frac{1}{2} T \sum_{n} \operatorname{Tr}\left\langle\hat{G}\left(\mathbf{0}, \mathbf{0} ; \omega_{n}+\nu_{m}\right) \hat{G}\left(\mathbf{0}, \mathbf{0} ; \omega_{n}\right)\right\rangle_{i m p}
$$

where $\omega_{n}=(2 n+1) \pi T$. Note that this representation of the spin correlator in terms of the $\hat{G}^{\prime}$ s is not unique, see section IV below.

We consider a single spin-degenerate electron band in a three-dimensional (3D) crystal and neglect the spin-orbit coupling. The electron Hamiltonian is written as $H=H_{0}+H_{i n t}$, where

$$
H_{0}=\int d^{3} \boldsymbol{r} \psi_{\alpha}^{\dagger}(\boldsymbol{r}) h_{0} \psi_{\alpha}(\boldsymbol{r})
$$

describes noninteracting electrons, with $\alpha=\uparrow, \downarrow$ being the spin projection, and

$$
h_{0}=-\frac{\nabla_{\boldsymbol{r}}^{2}}{2 m}+U(\boldsymbol{r})-\epsilon_{F} .
$$

Without loss of generality we assume an isotropic parabolic band. The impurity potential $U(\boldsymbol{r})$ is characterized by the correlator

$$
\left\langle U\left(\boldsymbol{r}_{1}\right) U\left(\boldsymbol{r}_{2}\right)\right\rangle_{i m p}=\frac{1}{2 \pi N_{F} \tau_{e l}} \delta\left(\boldsymbol{r}_{1}-\boldsymbol{r}_{2}\right),
$$

where $N_{F}$ is the density of states at the Fermi level, and $\tau_{e l}$ is the elastic mean free time.

In a weak-coupling BCS superconductor, the interaction Hamiltonian which describes s-wave singlet pairing has the following form:

$$
H_{i n t}=\frac{1}{2} \int d^{3} \boldsymbol{r} V_{\alpha \beta, \gamma \delta} \psi_{\alpha}^{\dagger}(\boldsymbol{r}) \psi_{\beta}^{\dagger}(\boldsymbol{r}) \psi_{\gamma}(\boldsymbol{r}) \psi_{\delta}(\boldsymbol{r})
$$

where $V_{\alpha \beta, \gamma \delta}=-(\lambda / 2)\left(i \sigma_{2}\right)_{\alpha \beta}\left(i \sigma_{2}\right)_{\gamma \delta}^{\dagger}$, and $\lambda>0$ is the coupling constant. Treating the pairing interaction in the mean-field approximation, the equation of motion for the matrix Green's function (4) can be written in the form

$$
\left(\begin{array}{cc}
-\partial_{\tau}-h_{0} & -\Delta(\boldsymbol{r}) \\
-\Delta^{*}(\boldsymbol{r}) & -\partial_{\tau}+h_{0}
\end{array}\right) \hat{G}\left(\boldsymbol{r}, \tau ; \boldsymbol{r}^{\prime}, \tau^{\prime}\right)=\hat{\tau}_{0} \delta\left(\boldsymbol{r}-\boldsymbol{r}^{\prime}\right) \delta\left(\tau-\tau^{\prime}\right)
$$

where $\hat{\tau}_{0}$ is the unity matrix in the Nambu space, and $\Delta(\boldsymbol{r})=\lambda F(\boldsymbol{r}, \tau ; \boldsymbol{r}, \tau)$ is the gap function. 
The spin correlator (7) will now be calculated using the exact eigenstates method, see e.g. Ref.12, which allows one to relate properties of the system in the superconducting state to those in the normal state. The key assumption is that the disorder-induced fluctuations of the gap function can be ignored:

$$
\Delta(\boldsymbol{r}) \rightarrow \Delta=\langle\Delta(\boldsymbol{r})\rangle_{i m p}
$$

According to Anderson's theorem, the average gap is not renormalized by nonmagnetic impurities, i.e. the value of $\Delta$ at any temperature is the same as in the clean case. We introduce the exact eigenstates and eigenvalues of the single-particle Hamiltonian with impurities:

$$
h_{0} \varphi_{a}(\boldsymbol{r})=\xi_{a} \varphi_{a}(\boldsymbol{r}), \quad \int d^{3} \boldsymbol{r}\left|\varphi_{a}(\boldsymbol{r})\right|^{2}=1
$$

where, according to the definition (9), the chemical potential is included in $\xi_{a}$. Then the Gor'kov equations (12) can be solved, with the result

$$
\hat{G}\left(\boldsymbol{r}, \boldsymbol{r}^{\prime} ; \omega_{n}\right)=\sum_{a} \hat{g}_{a}\left(\omega_{n}\right) \varphi_{a}(\boldsymbol{r}) \varphi_{a}^{*}\left(\boldsymbol{r}^{\prime}\right)
$$

where

$$
\hat{g}_{a}\left(\omega_{n}\right)=-\frac{i \omega_{n} \hat{\tau}_{0}+\xi_{a} \hat{\tau}_{3}+\Delta \hat{\tau}_{1}}{\omega_{n}^{2}+\xi_{a}^{2}+\Delta^{2}} .
$$

Substituting this in Eq. (17) we obtain, after the Matsubara frequency summation and the analytic continuation $i \nu_{m} \rightarrow \omega_{0}+i 0:$

$$
\begin{aligned}
\lim _{\omega_{0} \rightarrow 0} \frac{\operatorname{Im} K^{R}\left(\omega_{0}\right)}{\omega_{0}}= & -\frac{\pi}{2}\left\langle\sum_{a, b}\left|\varphi_{a}(\mathbf{0})\right|^{2}\left|\varphi_{b}(\mathbf{0})\right|^{2} \delta\left(E_{a}-E_{b}\right)\right. \\
& \left.\times\left(-\frac{\partial f}{\partial E_{a}}\right)\left(1+\frac{\xi_{a} \xi_{b}+\Delta^{2}}{E_{a} E_{b}}\right)\right\rangle_{i m p}
\end{aligned}
$$

where $f(x)=\left(e^{x / T}+1\right)^{-1}$ is the Fermi function, and $E_{a}=\sqrt{\xi_{a}^{2}+\Delta^{2}}$.

Next, we introduce the local DoS in the normal state:

$$
N(\boldsymbol{r}, \epsilon)=\sum_{a}\left|\varphi_{a}(\boldsymbol{r})\right|^{2} \delta\left(\epsilon-\xi_{a}\right),
$$

perform the disorder averaging in Eq. (17), and insert the result in the expression (1) for the relaxation rate:

$$
R=J^{2} N_{F}^{2} \int_{-\infty}^{\infty} d \omega\left(-\frac{\partial f}{\partial \omega}\right) \int_{-\infty}^{\infty} d \epsilon_{1} d \epsilon_{2} \Gamma\left(\epsilon_{1}, \epsilon_{2} ; \omega\right) \mathcal{R}\left(\epsilon_{1}-\epsilon_{2}\right) .
$$

The superconducting gap enters here only through the function $\Gamma$, which in the weak-coupling case has the form $\Gamma=\Gamma_{w c}$, with

$$
\begin{aligned}
\Gamma_{w c}\left(\epsilon_{1}, \epsilon_{2} ; \omega\right)= & \frac{\pi}{2}\left(1+\frac{\epsilon_{1} \epsilon_{2}+\Delta^{2}}{\epsilon_{1}^{2}+\Delta^{2}}\right) \frac{\sqrt{\epsilon_{1}^{2}+\Delta^{2}}}{\left|\epsilon_{1}\right|} \\
& \times\left[\delta\left(\epsilon_{1}-\epsilon_{2}\right)+\delta\left(\epsilon_{1}+\epsilon_{2}\right)\right] \delta\left(\omega-\sqrt{\epsilon_{1}^{2}+\Delta^{2}}\right)
\end{aligned}
$$

while all effects of impurities are contained in the local DoS correlator

$$
\mathcal{R}(\Omega)=\frac{1}{N_{F}^{2}}\langle N(\mathbf{0}, \epsilon+\Omega) N(\mathbf{0}, \epsilon)\rangle_{i m p} .
$$

In the normal state, we set $\Delta=0$ in Eq. (20) and obtain $R_{n}=\pi J^{2} N_{F}^{2} \mathcal{R}(0)$. To calculate the local DoS correlator, we write $N(\mathbf{0}, \epsilon)=-\left[G^{R}(\mathbf{0}, \mathbf{0} ; \epsilon)-G^{A}(\mathbf{0}, \mathbf{0} ; \epsilon)\right] / 2 \pi i$, where

$$
G^{R(A)}(\mathbf{0}, \mathbf{0} ; \epsilon)=\sum_{a} \frac{\left|\varphi_{a}(\mathbf{0})\right|^{2}}{\epsilon-\xi_{a} \pm i 0}
$$


are the exact retarded (advanced) Green's functions of the normal metal. Neglecting the difference between the local and the Fermi-level densities of states, we replace $\langle N(\mathbf{0}, \epsilon)\rangle_{i m p}=N_{F}$, and obtain

$$
\mathcal{R}(\Omega)=1+\delta \mathcal{R}(\Omega)
$$

where

$$
\begin{aligned}
\delta \mathcal{R}(\Omega)=\frac{1}{2 \pi^{2}} & \frac{1}{N_{F}^{2}} \operatorname{Re}\left[\left\langle G^{R}(\mathbf{0}, \mathbf{0} ; \epsilon+\Omega) G^{A}(\mathbf{0}, \mathbf{0} ; \epsilon)\right\rangle_{i m p}\right. \\
& \left.-\left\langle G^{R}(\mathbf{0}, \mathbf{0} ; \epsilon+\Omega)\right\rangle_{i m p}\left\langle G^{A}(\mathbf{0}, \mathbf{0} ; \epsilon)\right\rangle_{i m p}\right]
\end{aligned}
$$

represents the impurity vertex corrections to the product of the retarded and advanced Green's functions. The terms containing the averages of two retarded or two advanced Green's functions vanish. The subsequent steps are standard. Introducing the disorder-averaged Green's functions

$$
G^{R(A)}(\boldsymbol{k}, \epsilon)=\frac{1}{\epsilon-\xi_{\boldsymbol{k}} \pm i / 2 \tau_{e l}}
$$

where $\xi_{\boldsymbol{k}}=\boldsymbol{k}^{2} / 2 m-\epsilon_{F}$, the impurity ladder diagrams corresponding to $\delta \mathcal{R}$ can be summed, with the following result:

$$
\delta \mathcal{R}(\Omega)=\frac{\tau_{e l}}{\pi N_{F}} \operatorname{Re} \int \frac{d^{3} \boldsymbol{q}}{(2 \pi)^{3}} \frac{\Phi^{2}(\boldsymbol{q}, \Omega)}{1-\Phi(\boldsymbol{q}, \Omega)},
$$

where

$$
\begin{aligned}
\Phi(\boldsymbol{q}, \Omega) & =\frac{1}{2 \pi N_{F} \tau_{e l}} \int \frac{d^{3} \boldsymbol{k}}{(2 \pi)^{3}} G^{R}\left(\boldsymbol{k}+\frac{\boldsymbol{q}}{2}, \epsilon+\omega\right) G^{A}\left(\boldsymbol{k}-\frac{\boldsymbol{q}}{2}, \epsilon\right) \\
& =\left\langle\frac{1}{1-i \Omega \tau_{e l}+i \tau_{e l} \boldsymbol{v}_{F}(\boldsymbol{k}) \boldsymbol{q}}\right\rangle_{\hat{\boldsymbol{k}}},
\end{aligned}
$$

$\boldsymbol{v}_{F}(\boldsymbol{k})$ is the Fermi velocity, and the angular brackets denote the Fermi-surface averaging. At small $\Omega$ and $\boldsymbol{q}, \Phi(\boldsymbol{q}, \Omega) \simeq$ $1+i \Omega \tau_{e l}-D q^{2} \tau_{e l}$, where $D=v_{F}^{2} \tau_{e l} / 3$ is the diffusion coefficient. Therefore the impurity vertex corrections lead to a diffusion pole in the momentum integral on the right-hand side of Eq. (26). In 3D, due to the convergence of the integral at $q \rightarrow 0$, the dependence of $\delta \mathcal{R}$ on $\Omega$ is not singular and can be neglected. On the other hand, $\Phi(\boldsymbol{q}, \Omega)$ decays slowly at $q \rightarrow \infty$ :

$$
\Phi(\boldsymbol{q}, \Omega)=-\frac{i}{2 v_{F} q \tau_{e l}} \ln \frac{1-i \Omega \tau_{e l}+i v_{F} q \tau_{e l}}{1-i \Omega \tau_{e l}-i v_{F} q \tau_{e l}} \stackrel{q \rightarrow \infty}{\simeq} \frac{\pi}{2 v_{F} \tau_{e l}} \frac{1}{q}
$$

for a 3D spherical Fermi surface. Thus it is necessary to introduce the ultraviolet cutoff of the order of the Fermi momentum $k_{F}$ in the integral (26), which gives the following estimate:

$$
\delta \mathcal{R}(\Omega) \propto \frac{\tau_{e l}}{N_{F}} \int_{0}^{k_{F}} q^{2} d q \frac{1}{v_{F}^{2} \tau_{e l}^{2}} \frac{1}{q^{2}} \propto \frac{1}{\epsilon_{F} \tau_{e l}}
$$

and

$$
\mathcal{R}(\Omega)=1+O\left(\frac{1}{\epsilon_{F} \tau_{e l}}\right) .
$$

Although there is some enhancement of the local DoS correlator and, therefore, of the nuclear spin relaxation rate due to the diffusive motion of electrons, the magnitude of this effect in a weakly disordered $\left(\epsilon_{F} \tau_{e l} \gg 1\right) 3 \mathrm{D}$ metal turns out to be negligibly small. Similar conclusions have also been reached in Ref $\underline{13}$. Thus we finally arrive at the following result in the normal state:

$$
R_{n}=\pi J^{2} N_{F}^{2}
$$

which is known as the Korringa law $\frac{1}{1}$.

In the superconducting state, the integral of $\Gamma_{w c}$ on the right-hand side of Eq. (19) has a logarithmic divergency, whose origin can be traced to the square-root singularity at the gap edge in the BCS density of states. This singularity is smeared and the divergency is removed in the presence of magnetic impurities or if the gap is anisotropic 14 . In this work we focus on the gap smearing due to the strong-coupling effects in the Eliashberg formalism. 


\section{EXACT EIGENSTATES METHOD: STRONG COUPLING}

In this section, the exact eigenstates method is generalized to include the effects of electron-phonon interaction, see also Refs. 15,16 . The Hamiltonian is written as $H=H_{0}+H_{p h}+H_{e-p h}$, where $H_{0}$ describes noninteracting electrons and is given by Eq. (8),

$$
H_{p h}=\sum_{\boldsymbol{q}, j} \omega_{j}(\boldsymbol{q}) b_{\boldsymbol{q} j}^{\dagger} b_{\boldsymbol{q} j}
$$

is the Hamiltonian of free phonons, with $j$ labelling the phonon branches and $\omega_{j}(\boldsymbol{q})$ being the phonon dispersion (recall that $\hbar=1$ in our units), and

$$
H_{e-p h}=\sum_{\boldsymbol{k}_{1}, \boldsymbol{k}_{2}, j} g_{j}\left(\boldsymbol{k}_{1}, \boldsymbol{k}_{2}\right)\left(b_{\boldsymbol{q} j}+b_{-\boldsymbol{q} j}^{\dagger}\right) c_{\boldsymbol{k}_{1} \sigma}^{\dagger} c_{\boldsymbol{k}_{2} \sigma},
$$

describes the electron-phonon interaction $\left(\boldsymbol{q}=\boldsymbol{k}_{1}-\boldsymbol{k}_{2}\right)$. The electron-phonon vertex has the following properties: $g_{j}\left(\boldsymbol{k}_{1}, \boldsymbol{k}_{2}\right)=g_{j}^{*}\left(\boldsymbol{k}_{2}, \boldsymbol{k}_{1}\right)$ due to the hermiticity of $H_{e-p h}$, and $g_{j}\left(\boldsymbol{k}_{1}, \boldsymbol{k}_{2}\right)=g_{j}^{*}\left(-\boldsymbol{k}_{1},-\boldsymbol{k}_{2}\right)$ due to time-reversal symmetry. We assume unit volume and neglect the effects of impurities on the phonon spectrum. We also neglect the disorder effect on the electron-phonon vertices, which can be justified for long-wavelength acoustic phonons by the fact that electrons move with the lattice, including the impurity atoms, in order to preserve charge neutrality ${ }^{17,18}$. Although this argument cannot be extended to short-wavelength phonons, we make the usual assumption that the $g_{j}$ 's are nonrandom functions.

The matrix Green's function which includes the electron-phonon interaction but not disorder averaging satisfies the following equation:

$$
\hat{G}=\hat{G}_{0}+\hat{G}_{0} \hat{\Sigma}_{p h} \hat{G}
$$

Here $\hat{G}_{0}$ is the matrix Green's function of the disordered normal metal without phonons, whose coordinate representation can be obtained by setting $\Delta=0$ in Eq. (15):

$$
\hat{G}_{0}\left(\boldsymbol{r}, \boldsymbol{r}^{\prime} ; \omega_{n}\right)=-\sum_{a} \frac{i \omega_{n} \hat{\tau}_{0}+\xi_{a} \hat{\tau}_{3}}{\omega_{n}^{2}+\xi_{a}^{2}} \varphi_{a}(\boldsymbol{r}) \varphi_{a}^{*}\left(\boldsymbol{r}^{\prime}\right),
$$

and $\hat{\Sigma}_{p h}$ is the self-energy due to the electron-phonon interaction:

$$
\begin{array}{r}
\hat{\Sigma}_{p h}\left(\boldsymbol{k}, \boldsymbol{k}^{\prime} ; \omega_{n}\right)=-T \sum_{n^{\prime}} \sum_{\boldsymbol{k}_{1}, \boldsymbol{k}_{1}^{\prime}, j} \delta_{\boldsymbol{k}_{1}-\boldsymbol{k}_{1}^{\prime}, \boldsymbol{k}-\boldsymbol{k}^{\prime}} D_{j}\left(\boldsymbol{k}-\boldsymbol{k}_{1}, \omega_{n}-\omega_{n^{\prime}}\right) \\
\times g_{j}\left(\boldsymbol{k}, \boldsymbol{k}_{1}\right) g_{j}\left(\boldsymbol{k}_{1}^{\prime}, \boldsymbol{k}^{\prime}\right) \hat{\tau}_{3} \hat{G}\left(\boldsymbol{k}_{1}, \boldsymbol{k}_{1}^{\prime} ; \omega_{n^{\prime}}\right) \hat{\tau}_{3},
\end{array}
$$

where

$$
D_{j}\left(\boldsymbol{q}, \nu_{m}\right)=-\frac{2 \omega_{j}(\boldsymbol{q})}{\nu_{m}^{2}+\omega_{j}^{2}(\boldsymbol{q})}
$$

is the bare phonon propagator. The contributions to the self-energy from the diagrams with crossed phonon lines are neglected based on Migdal's theorem.

The self-energy (35) is still a random quantity. To make progress one has to assume that it can be averaged independently from the Green's functions, which amounts to replacing the random self-energy in the equation (33) by its disorder average:

$$
\hat{\Sigma}_{p h}\left(\boldsymbol{k}, \boldsymbol{k}^{\prime} ; \omega_{n}\right) \rightarrow \hat{\Sigma}_{p h}\left(\boldsymbol{k}, \omega_{n}\right)=\left\langle\hat{\Sigma}_{p h}\left(\boldsymbol{k}, \boldsymbol{k}^{\prime} ; \omega_{n}\right)\right\rangle_{i m p}
$$

This approximation is justified by the slow spatial variation of the self-energy compared to that of the electron Green's functions 15 . We use the subscript "ph" to emphasize that $\hat{\Sigma}_{p h}$ represents only the phononic part of the full self-energy (the latter contains also the impurity part: $\hat{\Sigma}=\hat{\Sigma}_{p h}+\hat{\Sigma}_{i m p}$ ). The Coulomb interaction can be included in a similar fashion. Although there are impurity vertex corrections to both the electron-phonon and the Coulomb vertices due to the diffusive motion of electrons, in a weakly disordered 3D superconductor those are small 15,19 . Thus, the average self-energy can be written as

$$
\begin{array}{r}
\hat{\Sigma}_{p h}\left(\boldsymbol{k}, \omega_{n}\right)=T \sum_{m} \frac{1}{N_{F}} \sum_{\boldsymbol{k}^{\prime}} \int_{0}^{\infty} d \Omega \alpha^{2} F\left(\boldsymbol{k}, \boldsymbol{k}^{\prime} ; \Omega\right) \frac{2 \Omega}{\nu_{m}^{2}+\Omega^{2}} \\
\times \hat{\tau}_{3} \hat{G}\left(\boldsymbol{k}^{\prime}, \omega_{n}-\nu_{m}\right) \hat{\tau}_{3},
\end{array}
$$


where

$$
\alpha^{2} F\left(\boldsymbol{k}, \boldsymbol{k}^{\prime} ; \Omega\right)=N_{F} \sum_{j}\left|g_{j}\left(\boldsymbol{k}, \boldsymbol{k}^{\prime}\right)\right|^{2} \delta\left[\Omega-\omega_{j}\left(\boldsymbol{k}-\boldsymbol{k}^{\prime}\right)\right]
$$

and $\hat{G}\left(\boldsymbol{k}, \omega_{n}\right)$ is the disorder-averaged Green's function of electrons.

For the exact eigenstates method to work we have to neglect the anisotropy of the electron-phonon interaction, which gives $\hat{\Sigma}_{p h}\left(\boldsymbol{k}, \omega_{n}\right)=\hat{\Sigma}_{p h}\left(\omega_{n}\right)$. Similar to the usual decomposition of the full self-energy, $\hat{\Sigma}=i \omega_{n}(1-Z) \hat{\tau}_{0}+\phi \hat{\tau}_{1} \underline{20}$, we represent its phononic part in the form

$$
\hat{\Sigma}_{p h}\left(\omega_{n}\right)=i \omega_{n}\left[1-Z_{p h}\left(\omega_{n}\right)\right] \hat{\tau}_{0}+\phi_{p h}\left(\omega_{n}\right) \hat{\tau}_{1},
$$

where $Z_{p h}$ and $\phi_{p h}$ are real and even functions of the Matsubara frequency. By analogy with the gap function $\Delta\left(\omega_{n}\right) \equiv \phi\left(\omega_{n}\right) / Z\left(\omega_{n}\right)$, one can define $\Delta_{p h}\left(\omega_{n}\right) \equiv \phi_{p h}\left(\omega_{n}\right) / Z_{p h}\left(\omega_{n}\right)$.

Replacing $\hat{\Sigma}_{p h}\left(\boldsymbol{k}, \boldsymbol{k}^{\prime}, \omega_{n}\right) \rightarrow \hat{\Sigma}_{p h}\left(\omega_{n}\right)$ in Eq. (33) we find that the electron Green's function before disorder averaging has the form (15), where $\hat{g}_{a}$ is now given by the following expression:

$$
\hat{g}_{a}\left(\omega_{n}\right)=-\frac{i \omega_{n} Z_{p h}\left(\omega_{n}\right) \hat{\tau}_{0}+\xi_{a} \hat{\tau}_{3}+\phi_{p h}\left(\omega_{n}\right) \hat{\tau}_{1}}{\omega_{n}^{2} Z_{p h}^{2}\left(\omega_{n}\right)+\xi_{a}^{2}+\phi_{p h}^{2}\left(\omega_{n}\right)} .
$$

Inserting this in Eq. (77), summing over the Matsubara frequencies and averaging with respect to disorder one obtains the expression (19) for the relaxation rate, with $\Gamma=\Gamma_{s c}$, where

$$
\begin{aligned}
& \Gamma_{s c}\left(\epsilon_{1}, \epsilon_{2} ; \omega\right)=\frac{\pi}{2} \operatorname{Tr}\left[\hat{\rho}\left(\epsilon_{1}, \omega\right) \hat{\rho}\left(\epsilon_{2}, \omega\right)\right], \\
& \hat{\rho}(\epsilon, \omega)=-\left.\frac{1}{\pi} \operatorname{Im} \frac{i \omega_{n} Z_{p h}\left(\omega_{n}\right) \hat{\tau}_{0}+\epsilon \hat{\tau}_{3}+\phi_{p h}\left(\omega_{n}\right) \hat{\tau}_{1}}{\omega_{n}^{2} Z_{p h}^{2}\left(\omega_{n}\right)+\epsilon^{2}+\phi_{p h}^{2}\left(\omega_{n}\right)}\right|_{i \omega_{n} \rightarrow \omega+i 0} .
\end{aligned}
$$

Using Eq. (29), the integrals over $\epsilon_{1}$ and $\epsilon_{2}$ can be calculated separately:

$$
\frac{1}{\pi} \int_{-\infty}^{\infty} d \epsilon \frac{i \omega_{n} Z_{p h}\left(\omega_{n}\right) \hat{\tau}_{0}+\epsilon \hat{\tau}_{3}+\phi_{p h}\left(\omega_{n}\right) \hat{\tau}_{1}}{\omega_{n}^{2} Z_{p h}^{2}\left(\omega_{n}\right)+\epsilon^{2}+\phi_{p h}^{2}\left(\omega_{n}\right)}=\frac{i \omega_{n} \hat{\tau}_{0}+\Delta\left(\omega_{n}\right) \hat{\tau}_{1}}{\sqrt{\omega_{n}^{2}+\Delta^{2}\left(\omega_{n}\right)}}
$$

Here we used the fact that the gap function is not renormalized by impurities: $\Delta_{p h}\left(\omega_{n}\right)=\Delta\left(\omega_{n}\right)^{20}$. Now one can perform the analytic continuation and obtain:

$$
\int_{-\infty}^{\infty} d \epsilon \hat{\rho}(\epsilon, \omega)=-\operatorname{Re} \frac{\omega \hat{\tau}_{0}+\Delta(\omega) \hat{\tau}_{1}}{\sqrt{\omega^{2}-\Delta^{2}(\omega)}}
$$

where the branch of the square root is chosen such that its real part has the same sign as $\omega$, and $\Delta(\omega)$ is the complex gap function for $\omega$ just above the real frequency axis ${ }^{20}$. Finally, using the normal-state relaxation rate (30), we obtain:

$$
\begin{aligned}
\frac{R_{s}}{R_{n}}= & \int_{-\infty}^{\infty} d \omega\left(-\frac{\partial f}{\partial \omega}\right) \\
& \times\left[\left(\operatorname{Re} \frac{\omega}{\sqrt{\omega^{2}-\Delta^{2}(\omega)}}\right)^{2}+\left(\operatorname{Re} \frac{\Delta(\omega)}{\sqrt{\omega^{2}-\Delta^{2}(\omega)}}\right)^{2}\right],
\end{aligned}
$$

which coincides with the clean-limit expression derived by Fibich in Ref $\stackrel{4}{4}$. This shows the absence of the impurity effects on the nuclear spin relaxation rate in a weakly disordered strong-coupling superconductor [up to the terms of the order of $\left.\left(\epsilon_{F} \tau_{e l}\right)^{-1}\right]$, which is the main result of this article.

Note that in general the disorder enters the expression for the relaxation rate through both the local DoS correlator $\mathcal{R}$ and $\Gamma_{s c}$, see Eq. (42). The latter depends on $Z_{p h}$ and $\phi_{p h}$, which are both renormalized by disorder. It is the smallness of nontrivial disorder-induced correlations of the local DoS in $3 \mathrm{D}$, i.e. the fact that $\mathcal{R}\left(\epsilon_{1}-\epsilon_{2}\right) \simeq 1$, that allows one to express the relaxation rate entirely in terms of the gap function $\Delta(\omega)$, which is not affected by disorder. 


\section{PERTURBATION THEORY IN THE LADDER APPROXIMATION}

As an additional check of our result (45), we now calculate the spin correlator (2) using a direct summation of the impurity vertex corrections in a standard diagram technique. While some of our intermediate results look similar to those of Ref. $\stackrel{7}{ }$, the final conclusion turns out to be qualitatively very different. We find, in agreement with the exact eigenstates method of section III, that the leading order correction to the nuclear spin relaxation rate resulting from impurity scattering is of the order of $\left(\epsilon_{F} \tau_{e l}\right)^{-1}$ in bulk superconductors.

We use the two-component Nambu operators (5) in the momentum representation:

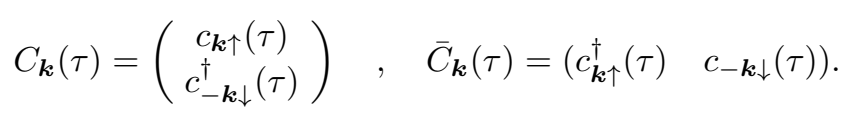

Then the spin-density operators (3) take the following form

$$
\begin{aligned}
S_{+}(\mathbf{0}, \tau) & =\frac{1}{2} \sum_{\boldsymbol{k}, \boldsymbol{k}^{\prime}} \bar{C}_{\boldsymbol{k} r}(\tau)\left(\hat{\tau}_{+}\right)_{r s} \bar{C}_{\boldsymbol{k}^{\prime} s}(\tau), \\
S_{-}(\mathbf{0}, 0) & =\frac{1}{2} \sum_{\boldsymbol{k}, \boldsymbol{k}^{\prime}} C_{\boldsymbol{k} r}(0)\left(\hat{\tau}_{-}\right)_{r s} C_{\boldsymbol{k}^{\prime} s}(0),
\end{aligned}
$$

where $r, s=1,2$ are the Nambu indices, and $\hat{\tau}_{ \pm}=\hat{\tau}_{1} \pm i \hat{\tau}_{2}$. The Hamiltonian of the system contains the electronphonon and the screened Coulomb interactions as well as the term describing the nonmagnetic impurity scattering. By applying the Wick theorem to the correlator (2), in which $S_{ \pm}$are given by the expressions (47), one finds in the clean case $K(\tau)=(1 / 2) \sum_{\boldsymbol{k} \boldsymbol{k}^{\prime}} \operatorname{Tr}\left[\hat{G}(\boldsymbol{k},-\tau)\left(i \hat{\tau}_{2}\right) \hat{G}\left(\boldsymbol{k}^{\prime},-\tau\right)\left(i \hat{\tau}_{2}\right)\right]$. In the presence of impurities we obtain the following form for the disorder-averaged $K\left(\nu_{m}\right)$, which includes only the second Born approximation impurity ladder diagrams 9,10 :

$$
\begin{array}{r}
K\left(\nu_{m}\right)=\frac{1}{2} \sum_{\boldsymbol{k}, \boldsymbol{q}} T \sum_{n} \operatorname{Tr}\left[\hat{G}\left(\boldsymbol{k}, \omega_{n}\right)\left(i \hat{\tau}_{2}\right) \hat{G}\left(\boldsymbol{k}+\boldsymbol{q},-\left(\omega_{n}+\nu_{m}\right)\right)\right. \\
\left.\times \hat{\Gamma}\left(\boldsymbol{q}, \omega_{n}, \omega_{n}+\nu_{m}\right)\right],
\end{array}
$$

where $\hat{G}$ is the matrix Green's function of electrons, disorder-averaged and fully dressed by all interactions. We assume a standard isotropic strong-coupling superconductor, for which

$$
\hat{G}\left(\boldsymbol{k}, \omega_{n}\right)=-\frac{i \omega_{n} Z\left(\omega_{n}\right) \hat{\tau}_{0}+\xi_{\boldsymbol{k}} \hat{\tau}_{3}+\phi\left(\omega_{n}\right) \hat{\tau}_{1}}{\omega_{n}^{2} Z^{2}\left(\omega_{n}\right)+\xi_{\boldsymbol{k}}^{2}+\phi^{2}\left(\omega_{n}\right)}
$$

with the momentum-independent renormalization function $Z\left(\omega_{n}\right)$ and the pairing self-energy $\phi\left(\omega_{n}\right)$, which are even functions of $\omega_{n}$. Both $Z$ and $\phi$ contain the effects of impurity scattering, which drop out of the gap function $\Delta\left(\omega_{n}\right)=\phi\left(\omega_{n}\right) / Z\left(\omega_{n}\right)$ in the isotropic single-band case ${ }^{20}$. The vertex function $\hat{\Gamma}$ satisfies the equation

$$
\begin{array}{r}
\hat{\Gamma}\left(\boldsymbol{q}, \omega_{n}, \omega_{n}+\nu_{m}\right)=i \hat{\tau}_{2}+\frac{1}{2 \pi N_{F} \tau_{e l}} \sum_{\boldsymbol{k}} \hat{\tau}_{3} \hat{G}\left(\boldsymbol{k}+\boldsymbol{q},-\omega_{n}-\nu_{m}\right) \\
\times \hat{\Gamma}\left(\boldsymbol{q}, \omega_{n}, \omega_{n}+\nu_{m}\right) \hat{G}\left(\boldsymbol{k}, \omega_{n}\right) \hat{\tau}_{3} .
\end{array}
$$

Note that Eq. (48) could also be obtained from the spin correlator (7), using the identity $\left(i \hat{\tau}_{2}\right) \hat{G}\left(\mathbf{0}, \mathbf{0},-\omega_{n}\right)\left(i \hat{\tau}_{2}\right)=$ $G\left(\mathbf{0}, \mathbf{0}, \omega_{n}\right)$. In the clean limit we recover from Eqs. (48) and (50) the Fibich's expression ${ }^{4}$ for the nuclear spin relaxation rate in isotropic single-band strong-coupling superconductors.

In order to solve Eq. (50), one represents the vertex function as a linear combination of the Pauli matrices in the Gor'kov-Nambu space: $\hat{\Gamma}=\sum_{i=0}^{3} \Gamma_{i} \hat{\tau}_{i}$, where $\Gamma_{i} \equiv \Gamma_{i}\left(\boldsymbol{q}, \omega_{n}, \omega_{n}+\nu_{m}\right)=(1 / 2) \operatorname{Tr}\left(\hat{\tau}_{i} \hat{\Gamma}\right)$, and finds a set of four coupled algebraic equations:

$$
\begin{aligned}
\Gamma_{0} & =\sum_{j} L_{0 j} \Gamma_{j} \\
\Gamma_{1} & =-\sum_{j} L_{1 j} \Gamma_{j} \\
\Gamma_{2} & =i-\sum_{j} L_{2 j} \Gamma_{j} \\
\Gamma_{3} & =\sum_{j} L_{3 j} \Gamma_{j},
\end{aligned}
$$


where $L_{i j} \equiv L_{i j}\left(\boldsymbol{q}, \omega_{n}, \omega_{n}+\nu_{m}\right)$, with $i, j=0,1,2,3$, are defined by

$$
L_{i j}=\frac{1}{2 \pi N_{F} \tau_{e l}} \frac{1}{2} \sum_{\boldsymbol{k}} \operatorname{Tr}\left[\hat{\tau}_{i} \hat{G}\left(\boldsymbol{k}+\boldsymbol{q},-\omega_{n}-\nu_{m}\right) \hat{\tau}_{j} \hat{G}\left(\boldsymbol{k}, \omega_{n}\right)\right] .
$$

One can see that $L_{10}=L_{01}, L_{20}=-L_{02}, L_{21}=-L_{12}, L_{30}=L_{03}, L_{31}=L_{13}, L_{32}=-L_{23}$, so that only ten out of sixteen $L_{i j}$ 's in Eq. (51) have to be computed. The sum over $\boldsymbol{k}$ in Eq. (52) is calculated approximately by using

$$
\sum_{\boldsymbol{k}} p\left(\xi_{\boldsymbol{k}}, \xi_{\boldsymbol{k}+\boldsymbol{q}}\right) \simeq \frac{N_{F}}{2} \int_{-1}^{1} d s \int_{-\infty}^{+\infty} d \xi p(\xi, \xi+Q(s))
$$

assuming that $p$ is decreasing fast enough with $\xi_{\boldsymbol{k}}$. Here $Q(s)=4 \epsilon_{F}\left(q / 2 k_{F}\right)\left(q / 2 k_{F}+s\right)$. In this way we find

$$
\begin{aligned}
& L_{00}=f_{-}\left(\frac{\Omega \Omega^{\prime}+\Phi \Phi^{\prime}}{D D^{\prime}}+1\right) \quad, \quad L_{01}=i f_{-} \frac{\Omega \Phi^{\prime}-\Omega^{\prime} \Phi}{D D^{\prime}}, \\
& L_{02}=-i f_{+}\left(\frac{\Phi}{D}+\frac{\Phi^{\prime}}{D^{\prime}}\right), \quad L_{03}=i f_{+}\left(\frac{\Omega}{D}+\frac{\Omega^{\prime}}{D^{\prime}}\right), \\
& L_{11}=f_{-}\left(\frac{\Omega \Omega^{\prime}+\Phi \Phi^{\prime}}{D D^{\prime}}-1\right) \quad, \quad L_{12}=f_{+}\left(\frac{\Omega}{D}-\frac{\Omega^{\prime}}{D^{\prime}}\right), \\
& L_{13}=f_{+}\left(\frac{\Phi}{D}-\frac{\Phi^{\prime}}{D^{\prime}}\right) \quad, \quad L_{22}=f_{-}\left(\frac{\Omega \Omega^{\prime}-\Phi \Phi^{\prime}}{D D^{\prime}}-1\right), \\
& L_{23}=f_{-} \frac{\Omega \Phi^{\prime}+\Omega^{\prime} \Phi}{D D^{\prime}}, \quad L_{33}=f_{-}\left(\frac{\Omega \Omega^{\prime}-\Phi \Phi^{\prime}}{D D^{\prime}}+1\right),
\end{aligned}
$$

where

$$
\begin{array}{ll}
\Omega=\omega_{n} Z\left(\omega_{n}\right), \quad \Phi=\phi\left(\omega_{n}\right), & D=\sqrt{\Omega^{2}+\Phi^{2}}, \\
\Omega^{\prime}=\left(\omega_{n}+\nu_{m}\right) Z\left(\omega_{n}+\nu_{m}\right), & \Phi^{\prime}=\phi\left(\omega_{n}+\nu_{m}\right), \quad D^{\prime}=\sqrt{\Omega^{\prime 2}+\Phi^{\prime}}
\end{array}
$$

and

$$
\begin{aligned}
& f_{+}=\frac{1}{32 \epsilon_{F} \tau_{e l}} \frac{1}{x} \ln \frac{x^{2}(x+1)^{2}+\left(D+D^{\prime}\right)^{2} / 16 \epsilon_{F}^{2}}{x^{2}(x-1)^{2}+\left(D+D^{\prime}\right)^{2} / 16 \epsilon_{F}^{2}} \\
& f_{-}=\frac{1}{16 \epsilon_{F} \tau_{e l}} \frac{1}{x}\left[\tan ^{-1} \frac{D+D^{\prime}}{4 \epsilon_{F} x(x-1)}-\tan ^{-1} \frac{D+D^{\prime}}{4 \epsilon_{F} x(x+1)}\right]
\end{aligned}
$$

with $x=q / 2 k_{F}$. We note that the relationship between our $f_{ \pm}$and $\left\langle f_{ \pm}\right\rangle$of Ref ? $^{7}\left\langle f_{+}\right\rangle=8 i f_{+},\left\langle f_{-}\right\rangle=-8 f_{-}$, with the additional difference that our $f_{ \pm}$contain $\left(D+D^{\prime}\right) / 4 \epsilon_{F}$, instead of $\left(D+D^{\prime}\right) / \epsilon_{F}$.

We solved the system (51) with the $L_{i j}$ 's defined by the expressions (53), using MAPLE. The result is

$$
\begin{aligned}
\Gamma_{0} & =\frac{f_{+}}{\left(1-2 f_{-}\right)^{2}+4 f_{+}^{2}}\left(\frac{\Phi}{D}+\frac{\Phi^{\prime}}{D^{\prime}}\right), \\
\Gamma_{1} & =-i \frac{f_{+}}{\left(1-2 f_{-}\right)^{2}+4 f_{+}^{2}}\left(\frac{\Omega}{D}-\frac{\Omega^{\prime}}{D^{\prime}}\right), \\
\Gamma_{2} & =i\left[1-\frac{f_{-}-2\left(f_{+}^{2}+f_{-}^{2}\right)}{\left(1-2 f_{-}\right)^{2}+4 f_{+}^{2}}\left(\frac{\Omega \Omega^{\prime}-\Phi \Phi^{\prime}}{D D^{\prime}}-1\right)\right], \\
\Gamma_{3} & =-i \frac{f_{-}-2\left(f_{+}^{2}+f_{-}^{2}\right)}{\left(1-2 f_{-}\right)^{2}+4 f_{+}^{2}} \frac{\Omega^{\prime} \Phi}{D D^{\prime}} .
\end{aligned}
$$

Inserting these into the vertex function in Eq. (48), we finally obtain:

$$
K\left(\nu_{m}\right)=-T \sum_{n=-\infty}^{\infty} A_{n} B_{n}
$$

where

$$
\begin{array}{r}
A_{n}=\frac{\omega_{n}\left(\omega_{n}+\nu_{m}\right)-\Delta\left(\omega_{n}\right) \Delta\left(\omega_{n}+\nu_{m}\right)}{\sqrt{\omega_{n}^{2}+\Delta^{2}\left(\omega_{n}\right)} \sqrt{\left(\omega_{n}+\nu_{m}\right)^{2}+\Delta^{2}\left(\omega_{n}+\nu_{m}\right)}}-1 \\
B_{n}=2 \pi \tau_{e l} N_{F} \sum_{\mathbf{q}}\left[f_{-}-2 \frac{f_{+}^{2}}{\left(1-2 f_{-}\right)^{2}+4 f_{+}^{2}}+2 \frac{f_{-}\left(f_{-}-2\left(f_{+}^{2}+f_{-}^{2}\right)\right)}{\left(1-2 f_{-}\right)^{2}+4 f_{+}^{2}}\right] .
\end{array}
$$


Clearly, in the limit $\tau_{e l} \rightarrow \infty$ only the first term in the square bracket in $B_{n}$ survives, and it is possible to integrate it over $\boldsymbol{q}$ analytically, with the result $\pi^{2} N_{F}^{2} \sqrt{\left(1+\sqrt{1+\rho^{2}}\right) / 2}$, where $\rho=\left(D+D^{\prime}\right) / \epsilon_{F}$. For all the terms in the sum over $n$ in Eq. (55) for which $A_{n}$ is nonzero [note that $\Delta\left(\omega_{n}\right) \simeq 0$ for $\left|\omega_{n}\right|$ greater than 10 times the maximum phonon frequency], $\rho$ can be set equal to zero. In this way one recovers Fibich's formula $\underline{4,5}$ for the relaxation rate, after the sum over $n$ is performed, followed by the analytic continuation $i \nu_{m} \rightarrow \omega_{0}+i 0$, in the limit $\omega_{0} \rightarrow 0$. We note that our $B_{n}$, containing the impurity vertex corrections, is different from the expression for the vertex corrections obtained by Choi and Mele ${ }^{7}$, which we have not been able to reproduce. An additional difference is that we calculate analytically the momentum integrals which were treated in Ref $\underline{\underline{7}}$ using some approximation.

Even for a finite $\tau_{e l}$ one can replace $\rho \rightarrow 0$, which makes it possible to integrate over $\boldsymbol{q}$ the second and third terms in the expression for $B_{n}$. Our final result for the nuclear spin relaxation rate in the superconducting state to the leading order in $\left(\epsilon_{F} \tau_{e l}\right)^{-1}$ is

$$
\begin{aligned}
R_{s}= & \pi J^{2} N_{F}^{2}\left(1+\frac{3 \pi}{16} \frac{1}{\epsilon_{F} \tau_{e l}}\right) \int_{-\infty}^{\infty} d \omega\left(-\frac{\partial f}{\partial \omega}\right) \\
& \times\left[\left(\operatorname{Re} \frac{\omega}{\sqrt{\omega^{2}-\Delta^{2}(\omega)}}\right)^{2}+\left(\operatorname{Re} \frac{\Delta(\omega)}{\sqrt{\omega^{2}-\Delta^{2}(\omega)}}\right)^{2}\right] .
\end{aligned}
$$

We see that the impurity vertex corrections turn out to be of the same order as the diagrams with crossed impurity lines, which we have neglected, and the ratio $R_{s} / R_{n}$ is therefore unaffected by impurity scattering at $\tau_{e l}^{-1} \ll \epsilon_{F}$. This condition is much weaker than $\ell \gg \xi_{0}$, i.e. $\tau_{e l}^{-1} \ll \Delta_{0}\left(\Delta_{0}\right.$ is the superconducting gap at zero temperature), found in Ref $\underline{\underline{7}}$.

\section{CONCLUSIONS}

We conclude that the answer to the question in the title is negative. Using two different techniques, the exact eigenstates method and the usual diagrammatic perturbation theory in the ladder approximation, we have shown that the contribution of nonmagnetic impurities to the nuclear spin relaxation rate in a bulk superconductor with isotropic pairing is of the order of $\left(\epsilon_{F} \tau_{e l}\right)^{-1}$, i.e. very small.

\section{Acknowledgments}

This work was supported by the Natural Sciences and Engineering Research Council (NSERC) of Canada.

1 Slichter C P 1990 Principles of Magnetic Resonance (Berlin: Springer-Verlag)

${ }^{2}$ Hebel L C and Slichter C P 1959 Phys. Rev. 1131504

3 Maki K and Fulde P 1965 Phys. Rev. 140 A1586

4 Fibich M 1965 Phys. Rev. Lett. 14561 (Erratum: Phys. Rev. Lett. 14 621)

5 Scalapino D J 1969 in Superconductivity vol. 1, ed. by R. D. Parks (New York: Marcel Dekker)

6 Eliashberg G M 1960 Sov. Phys. JETP 11696

7 Choi H-Y and Mele E J 1995 Phys. Rev. B 527549

8 Moriya T 1963 J. Phys. Soc. Jpn. 18516

9 Abrikosov A A, Gor'kov L P and Dzyaloshinskii I E 1963 Methods of Quantum Field Theory in Statistical Physics (New York: Dover Publishing)

10 Mahan G D 1990 Many-Particle Physics (New York: Plenum)

11 Schrieffer J R 1964 Theory of Superconductivity (New York: W. A. Benjamin)

12 De Gennes P G 1966 Superconductivity of Metals and Alloys (New York: W. A. Benjamin)

13 Shastry B S and Abrahams E 1994 Phys. Rev. Lett. 721933

14 Tinkham M 1996 Introduction to Superconductivity (New York: McGraw-Hill)

15 Keck B and Schmid A 1976 J. Low Temp. Phys. 24611

16 Belitz D 1987 Phys. Rev. B 351636

17 Tsuneto T 1961 Phys. Rev. 121402

18 Schmid A 1973 Z. Phys. 259421

19 Höhn T and Mitrović B 1994 Z. Phys. B 93163

20 Allen P B and Mitrović B 1982 in Solid State Physics vol. 37, ed. by F. Seitz et al. (New York: Academic Press) 